ORIGINAL ARTICLE

\title{
Superior results with eccentric compared to concentric quadriceps training in patients with jumper's knee: a prospective randomised study
}

\author{
P Jonsson, $\mathrm{H}$ Alfredson
}

Br J Sports Med 2005;39:847-850. doi: 10.1136/bjsm.2005.018630

See end of article for authors' affiliations

\section{Correspondence to:}

Per Jonsson, RPT, Sports Medicine Unit, University of Umeå, S-901 87 Umeå, Sweden; per.jonsson@ idrott.umu.se

Accepted 26 April 2005

\begin{abstract}
Background: A recent study reported promising clinical results using eccentric quadriceps training on a decline board to treat jumper's knee (patellar tendinosis).

Methods: In this prospective study, athletes (mean age 25 years) with jumper's knee were randomised to treatment with either painful eccentric or painful concentric quadriceps training on a decline board. Fifteen exercises were repeated three times, twice daily, 7 days/week, for 12 weeks. All patients ceased sporting activities for the first 6 weeks. Age, height, weight, and duration of symptoms were similar between groups. Visual analogue scales (VAS; patient estimation of pain during exercise) and Victorian Institute of Sport Assessment (VISA) scores, before and after treatment, and patient satisfaction, were used for evaluation.

Results: In the eccentric group, for $9 / 10$ tendons patients were satisfied with treatment, VAS decreased from 73 to $23(p<0.005)$, and VISA score increased from 41 to $83(p<0.005)$. In the concentric group, for $9 / 9$ tendons patients were not satisfied, and there were no significant differences in VAS (from 74 to $68, p<0.34$ ) and VISA score (from 41 to $37, p<0.34$ ). At follow up (mean 32.6 months), patients in the eccentric group were still satisfied and sports active, but all patients in the concentric group had been treated surgically or by sclerosing injections.

Conclusions: In conclusion, eccentric, but not concentric, quadriceps training on a decline board, seems to reduce pain in jumper's knee. The study aimed to include 20 patients in each group, but was stopped at the half time control because of poor results achieved in the concentric group.
\end{abstract}

T he aetiology of jumper's knee is not clearly understood but there is a general opinion that overuse of the patellar tendon is a triggering factor. ${ }^{1-3}$ Indeed, there are many theories about the pathogenesis of jumper's knee, but little scientific evidence. ${ }^{245}$ We believe it is important to use ultrasonography (US) for diagnosis to verify that the tendon structure is abnormal, since structural tendon changes do not necessarily cause tendon pain. ${ }^{67}$ Colour Doppler findings of neovessels in the area with structural tendon changes strengthen the assumption that the pain comes from the proximal patellar tendon. ${ }^{8-10}$

The pain mechanisms associated with this condition have not been fully clarified. ${ }^{5}$ An inflammatory component (chemical inflammation mediated via prostaglandin $\mathrm{E}_{2}$, $\mathrm{PGE}_{2}$ ) may be involved and anti-inflammatory medication is frequently administered. However, recent research using intra-tendinous microdialysis showed that there were no signs of chemical inflammation (normal $\mathrm{PGE}_{2}$ levels) in the chronic stage of the condition. ${ }^{11}$ Instead, based on recent findings from tendon biopsies, where substance P and CGRP immunoreactivity was found in blood vessels, a so called neurogenic inflammation is being considered. ${ }^{12}$

Jumper's knee (patellar tendinosis) is difficult to treat and sometimes brings the athlete's career to an end. There is no conservative or surgical treatment method that can be considered to be the treatment of choice. Results from surgery vary: studies with a poor study design have been demonstrated to be associated with good results, while studies with a good study design have been associated with poor results. ${ }^{13}$

In a recent uncontrolled study on a small group of patients, painful eccentric quadriceps training while standing on a decline board showed promising short term clinical results in athletes who were taken out of their athletic activity for the first 6 weeks of treatment. ${ }^{14}$ The aim of this randomised study was to compare the results of painful eccentric quadriceps training on a decline board with painful concentric quadriceps training on a decline board in a group of athletes who were taken out of their athletic activity for the first 6 weeks of treatment.

\section{METHODS \\ Participants}

Nineteen patellar tendons from 15 patients ( 13 men and two women, mean age 24.9 years) with a long duration (mean 17.4 months, range 8-72) of pain from the proximal patellar tendon and referred to the Sports Medicine Unit in Umeå, were included in the study. One orthopaedic surgeon (HA) examined all patients. The inclusion criteria were: pain in the proximal patellar tendon during or after patellar tendon loading activity, tenderness in the proximal patellar tendon during palpation, and structural tendon changes together with neovascularisation in the proximal patellar tendon at US and colour Doppler examination. US was performed with a linear transducer (Acuson Sequoia 512; Acuson, Mountain View, CA) at 8-13 MHz frequency. All US and colour Doppler examinations were carried out by the same experienced radiologist. Exclusion criteria were: a history of patellofemoral pain, surgical treatment of the patellar tendon, and knee arthrosis. The study protocol was approved by the ethical committee of the Medical Faculty, University of Umeå. Patient characteristics, previous treatment, and sporting activity are shown in table 1 .

Abbreviations: $\mathrm{PGE}_{2}$, prostaglandin $\mathrm{E}_{2}$; US, ultrasonography; VAS, visual analogue scale; VISA, Victorian Institute of Sport Assessment 
Table 1 Baseline data of the 15 patients (19 tendons) with jumper's knee

\begin{tabular}{lll}
\hline $\begin{array}{l}\text { Body } \\
\text { characteristics }\end{array}$ & $\begin{array}{l}\text { Concentric } \\
\text { training, } \mathbf{n}=9\end{array}$ & $\begin{array}{l}\text { Eccentric } \\
\text { training, } \mathbf{n = 1 0}\end{array}$ \\
\hline $\begin{array}{l}\text { Age, years } \\
\text { Height, } \mathrm{cm}\end{array}$ & $\begin{array}{l}24.1 \pm 6.4(17-32) \\
180.5 \pm 4.6(176-186)\end{array}$ & $\begin{array}{l}25.7 \pm 9.9(17-42) \\
176.6 \pm 6.6(165-182)\end{array}$ \\
$\begin{array}{l}\text { Weight, } \mathrm{kg} \\
\text { Duration of } \\
\text { symptoms, months }\end{array}$ & $\begin{array}{l}79.5 \pm 5.3(71-86) \\
19.6 \pm 20.3(8-72)\end{array}$ & $\begin{array}{l}74.8 \pm 7.8(65-84) \\
15.4 \pm 6(10-24)\end{array}$ \\
$\begin{array}{l}\text { Type of activity, } \mathrm{n} \\
\text { Running }\end{array}$ & 2 & \\
$\quad \begin{array}{l}\text { Soccer } \\
\text { Basketball }\end{array}$ & 3 & 5 \\
$\quad \begin{array}{l}\text { Floorball } \\
\text { Team handball }\end{array}$ & 1 & 2 \\
$\begin{array}{l}\text { Previous treatment, } \mathrm{n} \\
\text { Period of rest } \\
\text { NSAID }\end{array}$ & 5 & 3 \\
Physiotherapy & 3 & 4 \\
\hline \begin{tabular}{l} 
Mean \pm SD. Ranges are shown in parentheses. \\
\hline
\end{tabular} & 6 \\
\hline
\end{tabular}

\section{Randomisation procedure}

Sample size/power analysis

It was calculated that 20 patients in each group were needed for there to be an $80 \%$ chance of detecting a difference in the results of the treatments at the 0.05 significance level. The patients were randomly allocated to either eccentric or concentric quadriceps training while standing on a decline board.

\section{Treatment models}

There were two treatment models, eccentric quadriceps training while standing on a decline board and concentric quadriceps training while standing on a decline board. The eccentric training model was similar to that used in a recently published pilot study. ${ }^{14}$ Both training groups were given careful instructions by the same physiotherapist (PJ) on how to perform the training and increase the load. Both practical and hand written instructions were given. The training programme consisted of three sets of 15 repetitions each, performed twice a day, 7 days a week, for 12 weeks. The patients were told that muscle soreness during the first $1-$ 2 weeks was to be expected. The training was supposed to be painful, and when there was no pain in the patellar tendon during training, the load was to be increased to reach a new level of painful training by gradually add weights to a back pack. The patients themselves decided how much pain was acceptable. The patients were followed up by the physiotherapist (PJ) after 6 weeks of training. If there was no severe pain, the patients were told to start sport specific training and gradually return to their previous (before injury) sporting activity.

For both the eccentric and concentric training, the patients were instructed to keep the trunk in an upright position to minimise gluteal muscle activity while standing on a $25^{\circ}$ decline board. The purpose was to relax the calf muscles in order to increase the demands of the knee extensor muscles. ${ }^{14}$ If the patient had bilateral jumper's knee, the programme was completed separately for each leg.

\section{Eccentric quadriceps training}

The starting position for the eccentric quadriceps training was standing (trunk upright) on the $25^{\circ}$ decline board with the entire body weight on the injured leg. From that position the knee was slowly flexed to $70^{\circ}$ (fig lA,B). To return to the starting position, the other leg, or, if there were bilateral problems, the arms, were used. Concentric quadriceps activity was avoided as much as possible.
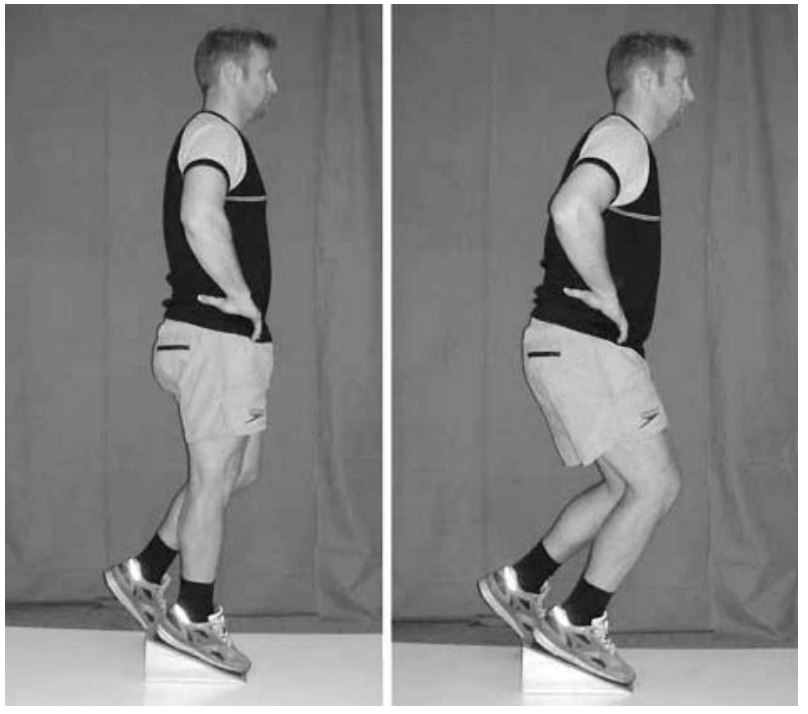

Figure 1 (A) Starting position for the eccentric quadriceps training on the $25^{\circ}$ decline board with the entire weight on the injured leg. From this position the knee was slowly flexed to $70^{\circ}$. (B) End position for eccentric quadriceps training. (Photographs are reproduced with permission.)

\section{Concentric quadriceps training}

The starting position for the concentric quadriceps training was standing (trunk upright) on the $25^{\circ}$ decline board with the entire body weight on the injured leg with the knee in $70^{\circ}$ flexion. From that position, the knee was slowly straightened to full extension (fig 2A,B). To return to the starting position, the other leg, or, if there were bilateral problems, the arms, were used. Eccentric quadriceps activity was avoided as much as possible.

\section{Outcome measures}

The outcome of the treatment was evaluated using a visual analogue scale (VAS) for pain, where the patients themselves recorded the amount of pain during their sporting activity on a $100 \mathrm{~mm}$ long pain scale. The amount of pain was recorded
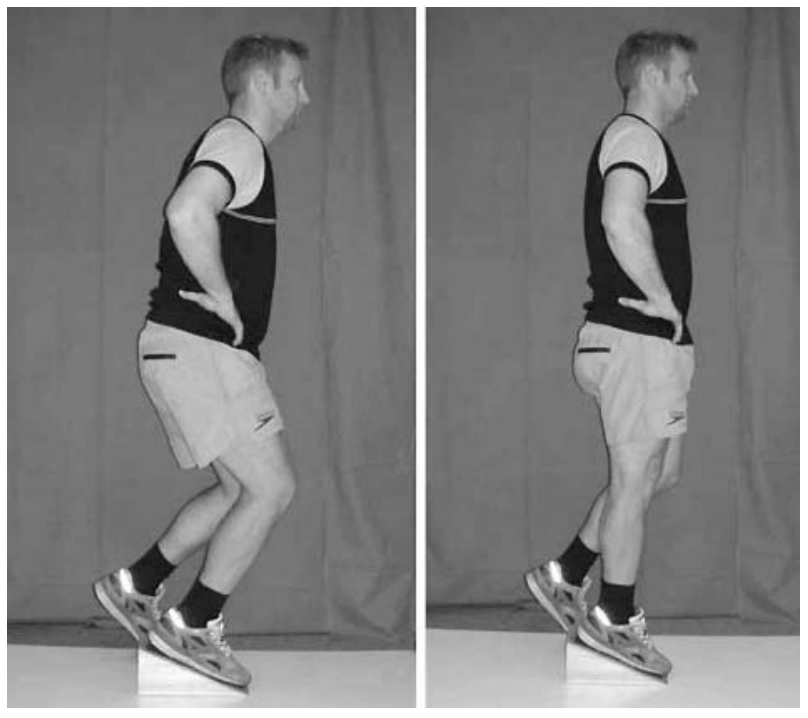

Figure 2 (A) Starting position for the concentric quadriceps training with the knee in $70^{\circ}$ flexion on the $25^{\circ}$ decline board. From this position, the knee was slowly straightened to full extension. (B) End position for concentric quadriceps training. (Photographs are reproduced with permission.) 
on a scale from 0 to 100 , where no pain is recorded as 0 and severe pain as 100. This was done at baseline (week 0 ) and at 12 weeks.

The Victorian Institute of Sport Assessment (VISA) score, which is a functional score that has been shown to be valid and reliable in patients with patellar tendinopathy, ${ }^{15}$ was assessed before and after treatment.

Patient satisfaction with treatment (satisfied or not satisfied) was also assessed.

\section{Statistical methods}

SPSS software for personal computers (version 11.5; SPSS, Chicago, IL) was used for the statistical analyses.

Subject characteristics are presented as means \pm SD and range.

Changes within the eccentric or concentric group between baseline and follow up at 12 weeks were tested using Wilcoxon signed ranks test. Differences between the concentric and eccentric group were tested using the MannWhitney $U$ test. A p value of less than 0.05 was considered significant.

\section{RESULTS}

Ten tendons from eight patients (seven men, one women, mean age $25.7 \pm 9.9$ years, range $17-42$ ) were randomised to the eccentric training programme and nine tendons from seven patients (six men, one women, mean age $24.1 \pm 6.4$ years, range 17-32) were randomised to the concentric training programme. There were no significant differences in age, weight, height, or duration of symptoms between the patients in the eccentric and concentric training groups.

\section{Drop outs}

Three patients (four tendons) in the concentric group did not continue the training programme after the 6 week follow up, due to severe tendon pain during and after training. Their mean VAS at the time of drop out was 75.

No VISA evaluation was done at the 6 week follow up because the patients had ceased athletic activity for the first 6 weeks.

At the 12 week follow up, the VAS score was significantly lower $(22 v 68, \mathrm{p}<0.01)$ and the VISA score was significantly higher $(83 v 37, \mathrm{p}<0.001)$ in the eccentric training group compared with the concentric training group. It should be noted that at the 12 week follow up, only five tendons remained in the concentric group due to drop outs after the 6 week follow up.

In the eccentric training group, there was a significant decrease in the VAS score at the 12 week follow up (baseline $72.7 \pm 16.2 \vee 22.5 \pm 26.4, \mathrm{p}<0.005)$. The VISA score increased significantly from $41.1 \pm 17.9$ at baseline to $83.3 \pm 23.4$ $(\mathrm{p}<0.005)$ at the 12 week follow up.

Seven patients (nine tendons) in the eccentric training group were satisfied with the treatment and had returned to their pre-injury sporting activity at the 12 week follow up. One patient was not satisfied with the results of the treatment and had not been able to return to previous sporting activity.

In the concentric training group, there was no significant difference in VAS at the 12 week follow up (baseline $74.3 \pm 16.6 v 68 \pm 18.5, \mathrm{p}<0.34)$. There was no significant difference in the VISA score at the 12 week follow up (baseline $40.7 \pm 16.3 \vee 37 \pm 4.6, \mathrm{p}<0.34$ ).

Three patients (four tendons) in the concentric training group were not satisfied with the results of the treatment at the 6 week follow up, while another four patients (five tendons) were not satisfied at the 12 week follow up. Altogether seven patients (nine tendons) in the concentric group were not satisfied and were unable to return to previous sporting activity after treatment. All nine tendons have now been treated with sclerosing injections or surgery.

Follow up a mean of 32.6 months after treatment showed that seven patients (nine tendons) in the eccentric group were still satisfied with the treatment. Six patients had returned to previous (before injury) activity, while one patient had stopped participating in his previous activity due to reasons other than patellar tendon pain. This patient was active in other knee loading recreational activities. Their mean VAS and VISA score were $18.3 \pm 21.6$ and $88.5 \pm 10.1$, respectively.

\section{DISCUSSION}

The short term results of this prospective randomised study in athletes with chronic painful jumper's knee showed that treatment with painful eccentric quadriceps training, but not with painful concentric quadriceps training, both while standing on a decline board, significantly reduced tendon pain during activity and improved function. These findings support the results from a recent non-randomised pilot study by Purdam et al. ${ }^{14}$ In our study, follow up 33 months after treatment showed that $7 / 8$ patients (9/10 tendons) in an eccentric training group were still satisfied and active in knee loading sports, while all patients in a concentric training group had been treated surgically or by sclerosing injections.

Due to severe pain after 6 weeks of training, there was a high frequency of drop outs (4/9 tendons) in the concentric group. This could possibly have impacted on the results, but because no patient in the concentric training group was satisfied with the result of the treatment, we considered it incorrect due to ethical reasons to recruit more patients into the study. The study aimed to include 20 patients in each group, but was stopped at the half time control.

\section{Diagnosis}

In the current study, the diagnosis of jumper's knee was established from clinical examination together with grey scale US and colour Doppler examination. By combining the clinical findings with the combined sonographic findings, the diagnosis is, most likely, accurate. ${ }^{89}$ In recent studies, neovessels in the area with structural tendon changes, visualised with the colour Doppler technique, have been demonstrated to be related to tendon pain during activity. ${ }^{8-10}$

\section{Aims}

The aim of our study was to compare eccentric with concentric quadriceps training. Therefore, we emphasised the use of exercises which, as far as possible, included either eccentric or concentric muscle contraction. However, especially for the concentric exercise, there was a short period of eccentric muscle contraction in the initial phase of the exercise.

\section{Evaluation}

To try to limit bias, treatment was evaluated by the patients themselves. The patients recorded the amount of patellar tendon pain during their sporting activity on a VAS and graded their tendon function on a VISA score. Patient satisfaction with treatment (satisfied/not satisfied) was also assessed.

\section{Treatment}

Jumper's knee is a troublesome condition to treat ${ }^{13}$ and frequently brings athletic careers to an end. Kettunen and coworkers found that $53 \%$ of patients with jumper's knee had to stop their sporting career due to knee pain. ${ }^{16}$ However, we cannot exclude that some of these patients also had other problems, such as cartilage defects. There is no treatment of choice for jumper's knee. Results of surgery vary and cannot be predicted, ${ }^{13}$ and no conservative method has been demonstrated to be outstanding. ${ }^{1}$ Eccentric calf muscle training has been shown to give very good clinical results 


\section{What is already known on this topic}

Jumper's knee is well known to be difficult to treat, both surgically and non-surgically. However, promising short term clinical results have been achieved recently using painful eccentric quadriceps training.

\section{What this study adds}

This short term study showed beneficial clinical effects with painful eccentric, but not painful concentric, quadriceps training on a decline board.

in patients with chronic mid-portion Achilles tendinosis, ${ }^{17}$ but similar good results have not been achieved with eccentric quadriceps training in patients with jumper's knee. However, it should be remembered that eccentric training does not produce good results in patients with Achilles tendon insertional problems, ${ }^{17}$ and jumper's knee is an insertional patellar tendon problem. Consequently, there seem to be differences in the response to eccentric training depending on where in the tendon the injury is located.

There are relatively few studies evaluating conservative treatment of jumper's knee, and to our knowledge, only two randomised studies comparing different models and evaluating function. ${ }^{18}{ }^{19}$ Studies by Stanish and Curwin ${ }^{20}$ and Jensen and Di Fabio ${ }^{21}$ reported promising clinical results using 6-8 weeks of pain free drop squat training. Unfortunately, no patient material or functional data were reported, which makes comparison difficult. Karlsson et $a l^{22}$ showed that $70 \%$ of patients with a partial patellar tendon rupture had excellent results after a combined concentric and eccentric pain free rehabilitation protocol. A randomised study by Cannell et al, ${ }^{18}$ using a 12 week programme of pain free drop squat or leg extension/curl exercise in patients involved in a variety of sports, found that both groups had significant reduction in pain. Although most of the patients returned to sport, only six of $19(32 \%)$ subjects were pain free after 12 weeks of training. In a recent prospective randomised controlled trial in volleyball players, it was demonstrated that a protocol consisting of painful eccentric decline squat exercises and a traditional pain free eccentric leg squat (on a $10 \mathrm{~cm}$ step) protocol were both effective in the treatment of tendon pain and sporting function. ${ }^{19}$ At the 3 month follow up, there were no differences in the results between the groups, which had significantly improved. However, over a 12 month period, the decline squat protocol produced a considerably greater likelihood of a 20 point or more improvement in VISA score. In our prospective randomised study, only painful eccentric, but not painful concentric, quadriceps training showed a significant improvement in the VAS and VISA scores after 12 weeks of training. Based on the results of the above studies, it seems that eccentric quadriceps training produces good clinical results. However, it should be remembered that only short term clinical results are reported; long term follow up studies are needed. The use of a decline board does not seem to significantly improve clinical outcome, but the groups of patients were small, and studies with larger numbers of subjects are needed to evaluate these results.

We cannot explain why painful eccentric, but not painful concentric, quadriceps training is associated with good clinical results. If the beneficial mechanisms are related to interference with nerves, maybe the "nerve damage" is caused more effectively during eccentric loading. Another possible explanation for the differences in the results might be that eccentric loading is associated with a better tissue response in terms of tissue repair mechanisms.

\section{CONCLUSION}

In the short term, treatment with painful eccentric quadriceps training, but not with painful concentric quadriceps training, while standing on a decline board, significantly reduced tendon pain during activity and improved function in athletes with chronic painful jumper's knee.

\section{Authors' affiliations}

P Jonsson, H Alfredson, Department of Surgical and Perioperative Science, Sports Medicine, Centre for Musculoskeletal Research, National Institute for Working Life, University of Umeå, 90187 Umeå, Sweden Competing interests: none declared

The patients detailed in this study agreed to their details being published

\section{REFERENCES}

1 Cook JL, Khan KM. What is the appropriate treatment for patellar tendinopathy? Br J Sports Med 2001;35(5):291-4.

2 Khan KM, Cook JL, Bonar F, et al. Histopathology of common overuse tendon conditions: update and implications for clinical management. Sports Med 1999;27:393-408.

3 Hamilton B, Purdam C. Patellar tendinosis as an adaptive process: a new hypothesis. Br J Sports Med 2004;38:758-61

4 Cook J, Feller JA, Bonar SF, et al. Abnormal tenocyte morphology is more prevalent than collagen disruption in asymptomatic athletes patellar tendons. $J$ Orthop Res 2004;22:334-8.

5 Khan KM, Cook JL, Maffulli N, et al. Where is the pain coming from in tendinopathy? It may be biochemical, not only structural, in origin. $\mathrm{Br} J$ Sports Med 2000;34:81-3

6 Cook JL, Khan KM, Harcourt PR, et al. Patellar tendon ultrasonography in asymptomatic active athletes reveals hypoechoic regions: a study of 320 tendons. Clin J Sports Med 1998;8:73-7.

7 Lian Ö, Holen KJ, Engebretsen L, et al. Relationship between symptoms of jumper's knee and the ultrasound characteristics of the patellar tendon among high level male volleyball players. Scand J Med Sci Sport 1996:6:291-6

8 Alfredson H, Öhberg L, Forsgren S. Is vasculo-neural ingrowth the cause of pain in chronic Achilles tendinosis? An investigation using ultrasonography and colour Doppler, immunohistochemistry, and diagnostic injections. Knee Surg Sports Traumatol Arthrosc 2003;11:334-8.

9 Cook JL, Malliaras P, De Luca J, et al. Neovascularization and pain in abnormal patellar tendons of active jumping athletes. Clin J Sport Med 2004; 14(5):296-9.

10 Alfredson $H$, Öhberg L. Neovascularisation in chronic painful patellar tendinosis - promising results after sclerosing neovessels outside the tendon challenges the need for surgery. Knee Surg Sports Traumatol Arthrosc 2004; 13:74-80.

11 Alfredson $\mathbf{H}$. In situ microdialysis in tendon tissue: high levels of glutamate, but not prostaglandin E2 in chronic Achilles tendon pain. Knee Surg Sports Traumatol Arthrosc 1999;7:378-81.

12 Forsgren S, Danielsson P, Alfredson $H$. Vascular NK-1R receptor occurrence in normal and chronic painful Achilles and patellar tendons. Studies on chemically unfixed as well as fixed specimens. Regul Pept 2005; 126:173-81.

13 Coleman BD, Khan KM, Maffulli N, et al. Studies of surgical outcome after patellar tendinopathy: clinical significance of methodological deficiencies and guidelines for future studies. Scand J Med Sci Sports 2000;10:2-11.

14 Purdam C, Jonsson P, Alfredson H, et al. A pilot study of the eccentric decline squat in the management of painful chronic patellar tendinopathy. $\mathrm{Br} J$ Sports Med 2004;38:395-7.

15 Visentini P, Khan KM, Cook JL, et al. The VISA score: an index of severity of jumper's knee. J Sci Med Sport 1998;1:22-8.

16 Kettunen JA, Kvist MK, Alanen E, et al. Long-term prognosis for jumper's knee in male athletes: a prospective follow-up study. Am J Sports Med 2002;30(5):689-92.

17 Fahlström M, Jonsson P, Lorentzon R, et al. Chronic Achilles tendon pain treated with eccentric calf-muscle training. Knee Surg Sports Traumatol Arthrosc 2003; 11:327-33

18 Cannell U, Taunton JE, Clement DB, et al. A randomized clinical trial of the efficacy of drop squat or leg extension/leg curl exercises to treat clinically diagnosed jumper's knee in athletes: pilot study. Br J Sports Med 2001;35:60-4.

19 Young MA, Cook JL, Purdam CR, et al. Eccentric decline squat protocol offers superior results at 12 months compared with traditional eccentric protocol for patellar tendinopathy in volleyball players. Br J Sports Med 2005;39:102-5.

20 Stanish WD, Curwin KS. Tendinitis. Its etiology and treatment. Toronto: Collamore Press, 1984.

21 Jensen K, Di Fabio RP. Evaluation of eccentric exercise in treatment of patellar tendinitis. Phys Ther 1989;69:211-16.

22 Karlsson J, Kälebo P, Goksör L-A, et al. Partial rupture of the patellar ligament. Am J Sports Med 1992;20:390-5. 